\title{
RESECCIÓN MIXTA LAPAROSCÓPICA Y ENDOSCÓPICA DE UN TUMOR DEL ESTROMA GASTROINTESTINAL DE LA UNIÓN GASTROESOFÁGICA*
}

\author{
Drs. Marcelo A. Beltrán S. ${ }^{1,2}$, Yamile Haito Ch. ${ }^{1}$, Ramón Díaz J. ${ }^{1}$, Oriana Urbina C. ${ }^{2}$, \\ Ints. Constanza Rodas N. ${ }^{3}$, Andrea De Balanzo A. ${ }^{3}$, Oliver Villa G. ${ }^{3}$
}

\author{
Servicio de Cirugía, Hospital de la Serena. \\ 2 Facultad de Medicina, Universidad Católica del Norte. \\ 3 Internos Facultad de Medicina, Universidad Católica del Norte. \\ La Serena, Chile.
}

\begin{abstract}
Resection of a gastrointestinal stromal tumor of the gastroesophagic junction using a mixed laparoscopic-endoscopic technique
\end{abstract}

Introduction: The ideal treatment for non-disseminated gastrointestinal stromal tumors (GIST) of the stomach is the local surgical resection. The laparoscopic approach has been validated showing its safety without compromising the oncological principles. The localization on the gastroesophagic junction is a specific situation on gastric GIST, mainly for submucosal tumors arising over the lesser curvature. Wedge resection of these tumors is technically demanding and has been associated to surgical complications and late development of stenosis and gastroesophageal reflux. For these reasons a mixed technique combining endoscopic and laparoscopic approaches has been developed. Herein we report a patient with an endophytic gastric GIST located on the gastroesophageal junction operated on with this technique. Clinical Case: A female 37-years old patient with a submucosal tumor of the gastroesophagic junction was submitted to laparoscopic surgery. During the surgical act the difficulty to continue with the laparoscopic resection became evident and the intragastric resection assisted with endoscopy was undertaken. The technique and the surgical outcomes are described. Conclusion. The intragastric approach for submucosal tumors located over the gastroesophagic junction is safe and relatively simple. This technique is widely described in the medical literature and its indications validated.

Key words: Gastrointestinal stromal tumor, GIST, laparoscopy, endoscopy.

\section{Resumen}

Introducción. El tratamiento de elección para los tumores localizados del estroma gastrointestinal (GIST) del estómago es la resección quirúrgica local. Se ha demostrado la seguridad de la técnica laparoscópica sin compromiso de los principios oncológicos. Un problema específico está constituido por los GIST de

*Recibido el 20 de octubre de 2013 y aceptado para publicación el 19 de abril de 2014.

Los autores no refieren conflictos de interés.

Correspondencia: Dr. Marcelo A. Beltrán S. beltran_01@yahoo.com 
la unión esófago-gástrica, principalmente los tumores de crecimiento intramural que se localizan en la curvatura menor. La resección en cuña de estos tumores no sólo es técnicamente demandante, sino que se asocia a complicaciones quirúrgicas y al desarrollo de estenosis de la unión gastroesofágica o a reflujo gastroesofágico por la extensa destrucción de la anatomía. Por estas razones se desarrolló una técnica mixta combinando el abordaje laparoscópico y endoscópico. En el presente reporte, describimos el caso de una paciente con un GIST gástrico endofítico de la unión esófago-gástrica que fue operado mediante esta técnica. Caso clínico: Paciente femenino de 37 años de edad, en la que se diagnostica un tumor de la unión esófago-gástrica. Ante la sospecha de un posible GIST gástrico se decide el abordaje laparoscópico, durante la cirugía se evidencia la dificultad de la resección laparoscópica por lo que se intenta la resección intragástrica asistida con endoscopia. Se describe la técnica y los resultados de la cirugía. Conclusiones. El abordaje intragástrico de tumores submucosos de la unión esófago-gástrica es seguro y relativamente simple. Esta técnica se encuentra ampliamente descrita en la literatura y sus indicaciones están validadas.

Palabras clave: Tumor del estroma gastrointestinal, GIST, laparoscopia, endoscopia.

\section{Introducción}

Los tumores del estroma gastrointestinal (GIST) se originan principalmente en el estómago $(65 \%)^{1,2}$. Cuando son sintomáticos, se presentan con hemorragia digestiva secundaria a ulceración de un tumor de crecimiento endofítico, o con síntomas derivados del efecto de masa, secundarios a un tumor de crecimiento exofítico ${ }^{3,4}$. El tratamiento de elección para los GIST gástricos localizados es la resección quirúrgica local con márgenes libres de tumor ${ }^{1-4}$. Se ha demostrado la seguridad de la técnica laparoscópica y los beneficios en términos de recuperación postoperatoria, sin compromiso de los principios oncológicos, para GIST gástricos menores a $7 \mathrm{~cm}$ que se operan mediante resección en cuña laparoscópica ${ }^{1,2}$. La primera resección laparoscópica de un GIST gástrico fue descrita en 1992 por Lukaszczyk y Preletz 5 . Desde entonces, para GIST gástricos seleccionados, la resección laparoscópica constituye la técnica de elección ${ }^{1,2,6-9}$. Un problema específico dentro de los tumores localizados en el estómago, está constituido por los GIST de la unión esófago-gástrica, principalmente los tumores de crecimiento intramural o predominantemente endofítico que se localizan en la curvatura menor. La resección en cuña de estos tumores no sólo es técnicamente demandante, sino que se asocia a complicaciones quirúrgicas y al desarrollo de estenosis de la unión gastroesofágica o a reflujo gastroesofágico por la extensa destrucción de la anatomía local ${ }^{1}$. Por estas razones los estudios de Tagaya ${ }^{10}$ y Ludwig ${ }^{11}$, publicados independientemente el año 2002 describiendo la resección intragástrica de estos tumores mediante una técnica mixta combinando el abordaje laparoscópico y endoscópico, han encontrado su lugar dentro del algoritmo de manejo quirúrgico de los GIST gástricos ${ }^{6-8}$. En el presente reporte, describimos el caso de una paciente con un GIST gástrico endofítico de la unión esófagogástrica que fue operado mediante la técnica mixta intragástrica.

\section{Caso clínico}

Paciente femenino de 37 años de edad, sin antecedentes patológicos conocidos, quien es estudiada por síntomas inespecíficos de dispepsia. Entre los estudios solicitados, la endoscopia alta describe un tumor submucoso de $3 \mathrm{~cm}$ en la unión esófagogástrica. El estudio se complementa con una tomografía computarizada (TC) abdominal que describe un tumor gástrico de aproximadamente $3 \times 2 \mathrm{~cm}$, localizado inmediatamente por debajo de la unión esófago-gástrica hacia la curvatura menor (Figura 1). Ante la sospecha de un posible GIST gástrico se decide el abordaje laparoscópico de la lesión. Inicialmente se diseca la unión esófago-gástrica desde el epiplón menor hasta identificar el esófago y el pilar derecho del diafragma. La exploración de la pared gástrica externa no revela el tumor, pero es posible palparlo con la pinza laparoscópica. Se decide continuar la exploración con una endoscopia alta, la cual identifica el tumor de la unión esófagogástrica. Ante la evidente dificultad de continuar con la cirugía por vía laparoscópica se decide intentar la resección intragástrica. Mediante visualización simultánea endoscópica-laparoscópica y utilizando los mismos puertos del abordaje laparoscópico se realizan dos incisiones en la pared gástrica anterior, triangulando la posición, uno hacia la curvatura mayor y el otro hacia la curvatura menor a nivel de la unión del tercio medio del estómago con el tercio superior. Se introduce una pinza dentada por la incisión de la curvatura mayor y una endograpadora lineal por la incisión de la curvatura menor (Figura 2). Se identifica el tumor por debajo de la unión esófago-gástrica, este es alargado $(3 \mathrm{~cm} \times 2 \mathrm{~cm})$, móvil y submucoso. Se toma el tumor con la pinza y con dos disparos de la endograpadora es resecado completamente (Figura 3). La pieza es retirada con un lazo a través del endoscopio (Figura 4). A continuación se extraen la pinza y la endograpadora y se suturan las incisiones en la pared gástrica con 

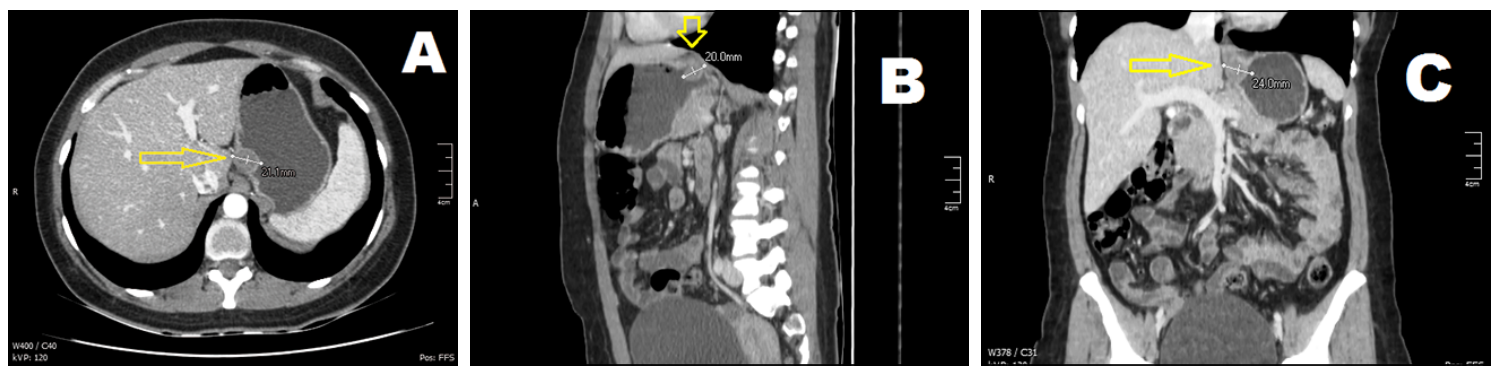

Figura 1. A. Tomografía computarizada (TC) abdominal. Corte transversal vista en fase arterial tardía del GIST gástrico que se presenta como un tumor ovalado, sólido y homogéneo. B. Vista sagital que muestra el GIST localizado adyacente a la unión esófago-gástrica. C. Corte coronal en fase venosa que muestra la localización del GIST en la unión esófago-gástrica.
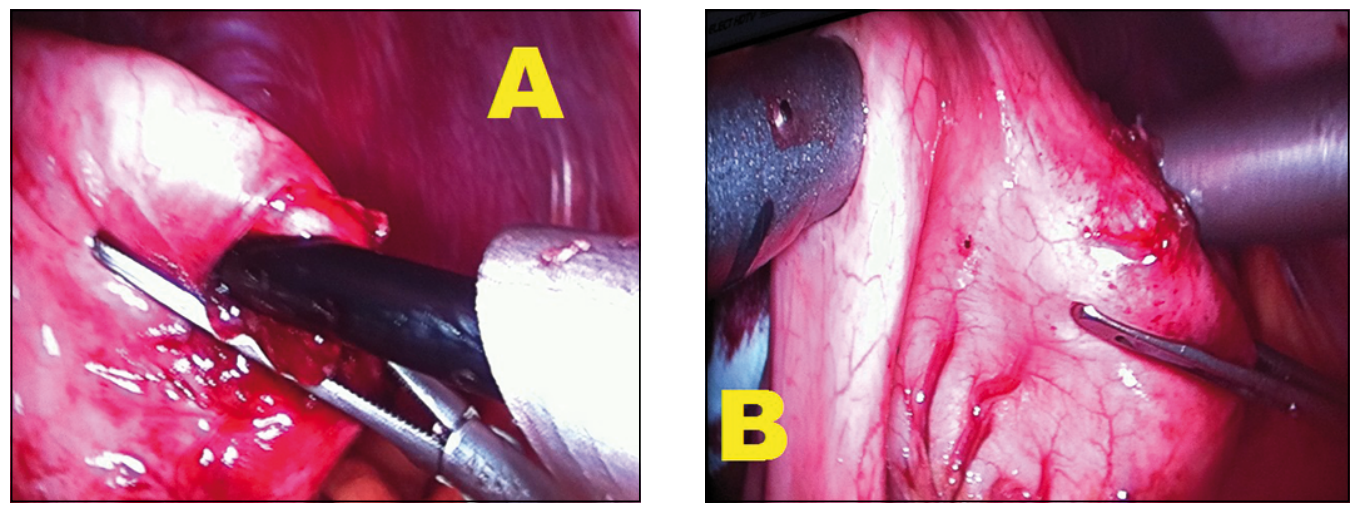

Figura 2. A. Puerto de $10 \mathrm{~mm}$ localizado en el flanco izquierdo del paciente. A través del mismo se introduce una pinza con dientes dentro del estómago bajo visión endoscópica simultánea. Con esta pinza se toma el tumor y se lo eleva para poder seccionarlo con la endograpadora. B. Puerto de $12 \mathrm{~mm}$ localizado en el flanco derecho del paciente. A través del mismo se introduce una endograpadora lineal con la cual se secciona el tumor submucoso.
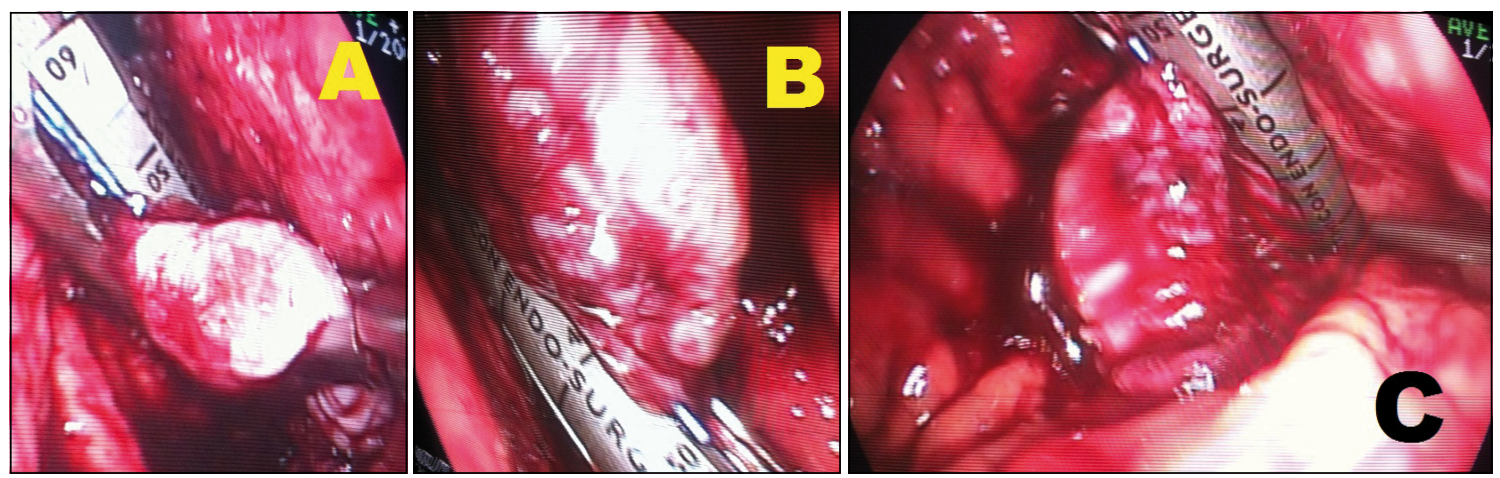

Figura 3. A. Imagen endoscópica que muestra la endograpadora tomando el tumor en el primer disparo de las grapas. B. Una vez seccionado el tumor con la primera carga de grapas, se lo toma nuevamente con la pinza endograpadora acomodándose para el segundo disparo. C. Segundo disparo de la endograpadora con el cual se completó la sección del tumor. 


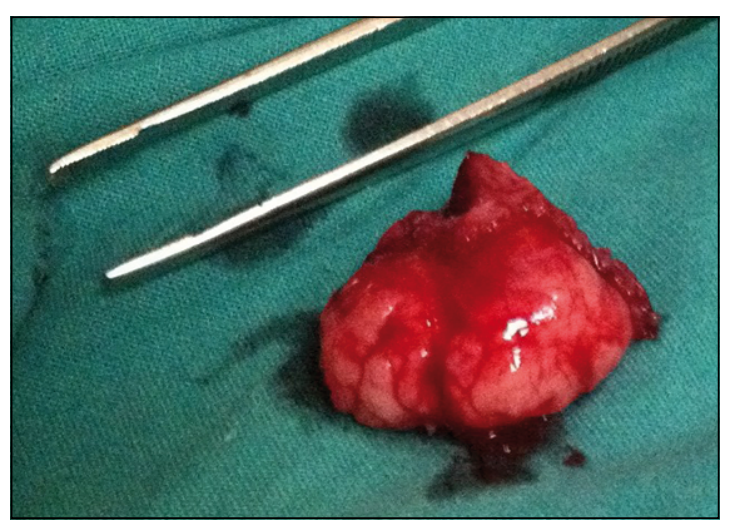

Figura 4. Tumor gástrico que muestra la línea de grapas en uno de sus bordes.

seda 1 (Figura 5). Nuevamente se realiza endoscopia confirmado el cierre de las incisiones gástricas bajo visión directa. Finalmente se realiza prueba neumática la cual es negativa para filtraciones. En el mismo procedimiento se realiza colecistectomía laparoscópica debido a que la paciente tenía múltiples cálculos en su vesícula. No se dejaron drenajes. La Figura 6 muestra un esquema de la posición de los cirujanos, de los puertos laparoscópicos en la paciente, de los monitores y del endoscopio. La paciente se realimentó a las $24 \mathrm{~h}$ y fue dada de alta $48 \mathrm{~h}$ después de la cirugía. La biopsia reveló un tumor que medía $3 \mathrm{~cm} \times 2,5 \mathrm{~cm} \times 1 \mathrm{~cm}$, con un margen libre de tumor entre 6 y $9 \mathrm{~mm}$. La tinción inmunohistoquímica fue positiva para CD117, CD34 y DOG-1. La actina de músculo liso $\alpha$, desmina, vimentina y $\mathrm{S}-100$ fueron negativos. El Ki67 fue positivo en menos de 1\% de las células neoplásicas y se encontraron 1 a 0 mitosis por 50 campos de aumento mayor. Por lo tanto, se consideró como un GIST con bajo riesgo de malignidad.

\section{Discusión}

El desarrollo de la cirugía laparoscópica y otros procedimientos mínimamente invasivos para la resección de ciertos tumores tales como los GIST, los que por sus características propias son susceptibles de ser sometidos a estas técnicas, ha llevado a la definición de protocolos y abordajes específicos basados en la localización del tumor dentro del tracto digestivo, principalmente cuando estos se localizan en el estómago ${ }^{7,8}$. Un problema especial lo constituyen los tumores que se encuentran en la unión esófago-gástrica o inmediatamente por debajo de ella y principalmente cuando estos son submucosos o de crecimiento predominantemente endofítico. En

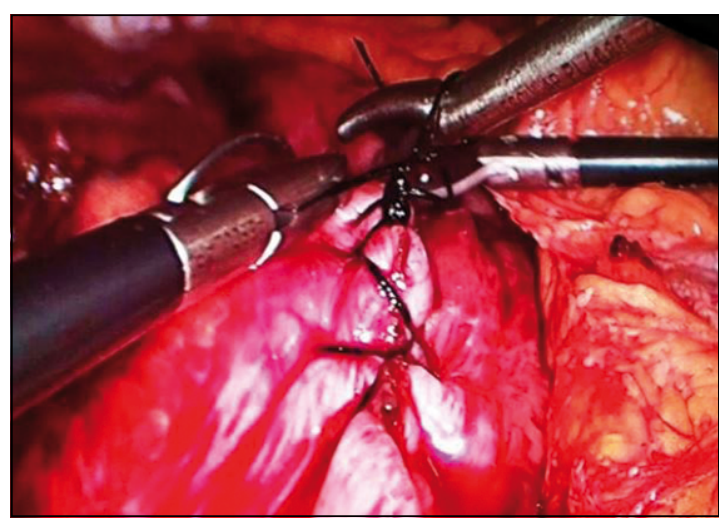

Figura 5. Sutura laparoscópica de las incisiones para las pinzas en la pared gástrica.

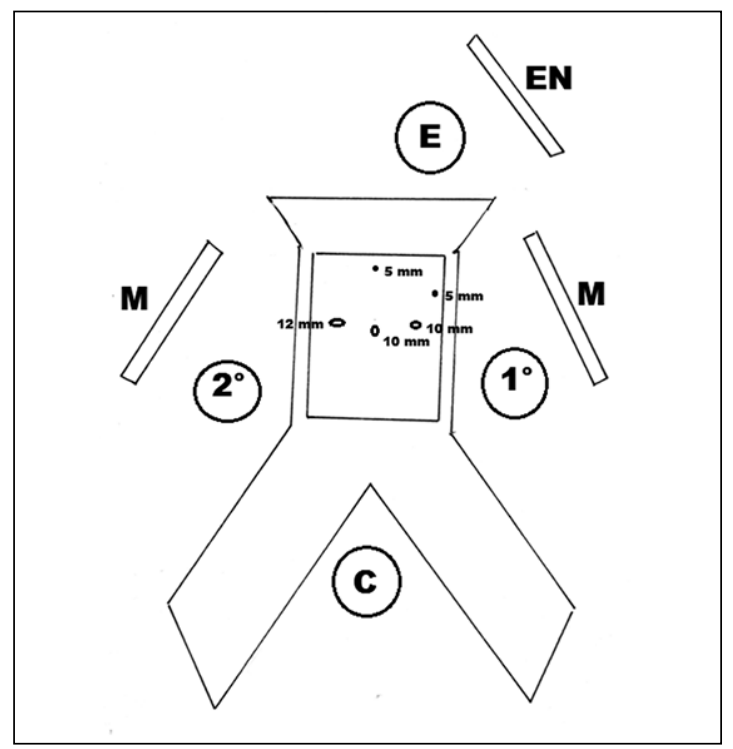

Figura 6. Paciente en posición francesa. C: Cirujano. $1^{\circ}$ : Primer ayudante. $2^{\circ}$ : Segundo ayudante y camarógrafo. E: Endoscopista. M: Monitores laparoscópicos. EN: Endoscopio. La disposición de los puertos laparoscópicos es estándar para toda cirugía de la unión gastroesofágica: $5 \mathrm{~mm}$ epigástrico, $5 \mathrm{~mm}$ subcostal izquierdo sobre la línea axilar media, $10 \mathrm{~mm}$ flanco izquierdo, $10 \mathrm{~mm}$ umbilical (cámara) y $12 \mathrm{~mm}$ flanco derecho.

estos casos la resección laparoscópica mediante las técnicas habituales, resección en cuña o resección segmentaria, son técnicamente difíciles o se asocian a complicaciones y compromiso de la función del órgano ${ }^{1}$. Por estas razones los procedimientos mixtos combinando el abordaje laparoscópico y la resección intragástrica asistida con endoscopia intraoperatoria, descritas independientemente el año 2002 
Tabla 1. Clasificación de Privette para GIST gástricos ${ }^{7}$

\begin{tabular}{|cll|}
\hline Tipo & Localización del GIST & Abordaje quirúrgico \\
I & Fondo o curvatura mayor & Resección en cuña \\
II & Pre-pilórico o antro & Gastrectomía distal \\
II & Curvatura menor o unión esófago-gástrica & Resección intragástrica \\
\hline
\end{tabular}

por Ludwig y Tagaya, han encontrado su lugar en el algoritmo del manejo de los tumores localizados en la unión esófago-gástrica ${ }^{6-8}$. El año 2008, Privette y cols $^{7}$, describieron una clasificación simple de los GIST gástricos de acuerdo a su localización en la pared gástrica y de acuerdo a ella propusieron el abordaje quirúrgico laparoscópico apropiado asociado a control endoscópico. El abordaje intragástrico encuentra su lugar en el caso de los GIST Tipo III, localizados en la curvatura menor o en la región de la unión esófago-gástrica (Tabla 1). Existen múltiples publicaciones constituidas por casos clínicos o pequeñas series de casos que han demostrado la seguridad y validez de este abordaje o de sus variaciones ${ }^{10-20}$. Recientemente, este procedimiento también se ha descrito mediante el uso de un solo puerto con la técnica Single Incision Laparoscopic Surgery (SILS) con buenos resultados ${ }^{21-24}$.

Hasta épocas recientes, en nuestra institución el diagnóstico y tratamiento de los GIST se efectuaba bajo condiciones de urgencia o en forma incidental $^{3,4,25-27}$. Actualmente con la amplia disponibilidad de estudios de imagen, la mayoría de los GIST son diagnosticados previamente a la cirugía y su abordaje planificado. Tal como se describe en la literatura, la localización más frecuente de los GIST en nuestros pacientes es el estómago ${ }^{3,4,26,27}$. La mayoría de los GIST gástricos operados en nuestro servicio han sido abordados mediante laparotomía realizando una gran variedad de procedimientos de acuerdo al tamaño y localización del tumor, incluyendo resección en cuña, gastrectomía distal, gastrectomía total y resección intragástrica a través de gastrotomía sobre la cara anterior del estómago. Anecdóticamente, en una oportunidad durante una gastrectomía tubular laparoscópica resecamos un GIST de crecimiento exofítico localizado en la cara posterior del antro gástrico, el cual fue incluido en la pieza quirúrgica con buenos resultados oncológicos ${ }^{27}$. El caso de la paciente descrita en este reporte fue planificado para abordaje laparoscópico con apoyo endoscópico, durante la cirugía, al comprobarse que no se podría llevar a cabo la resección del tumor mediante una cuña gástrica se decidió intentar el abordaje intragástrico descrito. El procedimiento fue relativamente simple y los resultados en términos de recuperación postoperatoria y de resección oncológica fueron excelentes.

\section{Conclusiones}

El abordaje intragástrico de tumores submucosos de la unión esófago-gástrica es seguro y relativamente simple. Esta técnica se encuentra ampliamente descrita en la literatura y sus indicaciones están validadas.

\section{Referencias}

1. Granger SR, Rollins MD, Mulhivill SJ, Glasgow RE. Lessons learned from laparoscopic treatment of gastric and gastroesophageal junction stromal tumors. Surg Endosc. 2006;20:1299-304

2. Goh BK, Chow PK, Chok AY, Chan WH, Chung YF, Ong HS, et al. Impact of the introduction of laparoscopic wedge resection as a surgical option for suspected small/medium-sized gastrointestinal stromal tumors of the stomach on perioperative and oncologic outcomes. J Gastrointest Surg. 2010;34:1847-52.

3. Beltrán MA, Vicencio AO, Barra MM, Contreras MA, Wilson CS, Cruces KS. Resultados del tratamiento quirúrgico de los tumores del estroma gastrointestinal (GIST) en la IV Región de Chile. Rev Chil Cir. 2011;63:290-6.

4. Beltrán MA, Pujado B, Pozo C, Méndez P, Barrera R, Mora R. Tumor gástrico gigante del estroma gastrointestinal. Gastroenterol Latinoam. 2007;18:378-82.

5. Lukaszczyk JJ, Preletz RJ. Laparoscopic resection of benign stromal tumor of the stomach. J Laparoendosc Surg. 1992;2:331-4.

6. Song KY, Kim SN, Park CH. Tailored- approach of laparoscopic wedge resection for treatment of submucosal tumor near the esophagogastric junction. Surg Endosc. 2007;21:2272-6.

7. Privette A, McCahill L, Borrazzo E, Single RM, Zubarik R. Laparoscopic approaches to resection of suspected gastric gastrointestinal stromal tumors based on tumor location. Surg Endosc. 2008;22:487-94.

8. Sasaki A, Koeda K, Obuchi T, Nakajima J, Nishizuka $\mathrm{S}$, Terashima M, et al. Tailored laparoscopic resection 
for suspected gastric gastrointestinal stromal tumors. Surgery 2010;147:516-20.

9. Blay JY, Bonvalot S, Casali P, Choi H, Debiec-Richter M, Dei Tos AP, et al. Consensus meeting for the management of gastrointestinal stromal tumors. Report of the GIST Consensus Conference of 20-21 March 2004, under the auspices of ESMO. Annals of Oncology 2005;16:566-78.

10. Tagaya N, Mikami H, Kogure H, Kubota K, Hoyosa Y, Nagai H. Laparoscopic intragastric stapled resection of gastric submucosal tumors located near the esophagogastric junction. Surg Endosc. 2002;16:177-9.

11. Ludwig K, Willhelm L, Scharlau U, Amtsberg G, Bernhardt J. Laparoscopic- endoscopic rendezvous resection of gastric tumors. Surg Endosc. 2002;16:1561-5.

12. Hiki N, Yamamoto Y, Fukunaga T, Yamaguchi T, Nunobe S, Tokunaga M, et al. Laparoscopic and endoscopic cooperative Surgery for gastrointestinal stromal tumor dissection. Surg Endosc. 2008;22:1729-35.

13. Abe N, Takeuchi H, Yanagida O, Masaki T, Mori T, Sugiyama M, et al. Endoscopic full-thickness resection with laparoscopic assistance as hybrid NOTES for gastric submucosal tumor. Surg Endosc. 2009;23:1908-13.

14. Uchikoshi F, Ito $T$, Nishida $T$, Kitagawa $T$, Endo $\mathrm{S}$, Matsuda H. Laparoscopic intragastric resection of gastric stromal tumor located at the esophagocardiac junction. Surg Laparosc Endosc Percutan Tech. 2004;14:1-4.

15. Shim JH, Lee HH, Yoo HM, Jeon HM, Park CH, Kim $\mathrm{JG}$, et al. Intragastric approach for submucosal tumors located near the Z-line: A hybrid laparoscopic and endoscopic technique. J Surg Oncol. 2011;104:312-5.

16. Warsi AA, Peyser PM. Laparoscopic resection of gastric GIST and benign gastric tumors: Evolution of a new technique. Surg Endosc. 2010;24:72-8.

17. Tsujimoto H, Yaguchi Y, Kumano I, Takahata R, Ono $\mathrm{S}$, Hase K. Successful gastric submucosal tumor resection using laparoscopic and endoscopic cooperative surgery. World J Surg. 2012;36:327-30.
18. Wilhelm D, Delius SV, Burian M, Schneider A, Frimberger E, Meining A, et al. Simultaneous use of laparoscopy and endoscopy for minimally invasive resection of gastric subepithelial masses: Analysis of 93 interventions. World J Surg. 2008;32:1021-8.

19. Walsh RM, Ponsky J, Brody F, Matthews BD, Heniford BT. Combined endoscopic/laparoscopic intragastric resection of gastric stromal tumors. J Gastrointest Surg. 2003; 7:386-92.

20. Tagaya N, Mikami H, Kubota K. Laparoscopic resection of gastrointestinal mesenchymal tumors located in the upper stomach. Surg Endosc. 2004;18:1469-74.

21. Pross M, Wolff S, Nestler G, Schubert D, Kahl S, Lippert $\mathrm{H}$. A technique for endo-organ resection of gastric wall tumors using one intragastric trocar. Endoscopy 2003;35:613-5.

22. Sahm M, Pross M, Lippert H. Intraluminal resection of gastric tumors using intragastric trocar technique. Surg Laparosc Endosc Percutan Tech. 2011;21:169-72.

23. De Vogelaere K, Van De Winkel N, Simoens C, Delvaux G. Intragastric SILS for GIST, a new challenge in oncologic Surgery: First experiences. Anticancer Res. 2013;33:3359-63.

24. Na JU, Lee SI, Noh SM. The single incision laparoscopic Intragastric wedge resection of gastric submucosal tumors. J Gastric Cancer 2011;11:225-9.

25. Beltrán MA, Cruces KS. Primary tumors of jejunum and ileum as a cause of intestinal obstruction: A case control study. Int J Surg. 2007;5:183-91.

26. Beltrán MA, Cortés VJ, Tapia RA, Larraín CT, Jaramillo LR, Oyarzún JS, y cols. Desarrollo de resistencia secundaria a imatinib y tratamiento quirúrgico de un GIST gástrico maligno. Gastroenterol Latinoam. 2012;23:84-8.

27. Beltrán MA, Pujado B, Méndez PE, González FJ, Margulis DI, Contreras MA, et al. Gastric gastrointestinal stromal tumor (GIST) incidentally found and resected during laparoscopic sleeve gastrectomy. Obes Surg. 2010;20:393-6. 